Vietnam Journal of Mechanics, VAST, Vol.40, No. 4 (2018), pp. 377 -385

DOI: https://doi.org/10.15625/0866-7136/12936

\title{
EFFECTIVE CONDUCTIVITY OF ISOTROPIC COMPOSITE WITH KAPITZA THERMAL RESISTANCE
}

\author{
Nguyen Trung Kien ${ }^{1, *}$, Nguyen Van Luat ${ }^{2}$, Pham Duc Chinh ${ }^{3}$ \\ ${ }^{1}$ University of Transport and Communications, Hanoi, Vietnam \\ ${ }^{2}$ Hanoi University of Industries, Vietnam \\ ${ }^{3}$ Institute of Mechanics, VAST, Hanoi, Vietnam \\ *E-mail: ntkien@utc.edu.vn \\ Received August 07, 2018
}

\begin{abstract}
A simple method is introduced for computing the effective conductivity of isotropic composite with imperfect interface. Based on the doubly-coated circle assemblage model, one can determine the effective thermal conductivity of the composite. The application of this model to the composite with imperfect interface of the Kapitza's type is proposed. The results obtained were compared with the Fast Fourier Transform simulation and the equivalent inclusion approximation in 2D show the effectiveness of the methods.
\end{abstract}

Keywords: effective conductivity, imperfect interface, Kapitza thermal resistance.

\section{INTRODUCTION}

Understanding of the effects of the microstructural characteristics on effective properties of composite has been the subject of numerous works in the framework of the homogenization theory. In the literature, most research has focused on the idealized case of perfect interface contact. However many experimental results $[1,2]$ indicate that the effective thermal conductivity of composites can be affected by a thermal resistance at the interface between the individual components. The theoretical analyses of this problem were conducted by Benveniste and Miloh [3], Hasselman and Johnson [4], Dunn and Taya [5], Torquato and Rintoul [6]. An important point to all these works is that the effective conductivity of the composites depends on the size of the inclusion. The purpose of the present paper is to propose a simple method to compute the effective thermal conductivity for isotropic composite with Kapitza interface resistance in two-dimensional space. The paper is organized as follows. Section 2 presents a multi-coated sphere assemblage model. Section 3 will apply this model for materials with imperfect interface of Kapitza type in which the intermediate phase is very thin and the conductivity depends on the

(C) 2018 Vietnam Academy of Science and Technology 
thinness of the phase. Section 4 presents the numerical calculation from Fourier Transform method (FFT) when replacing the inclusion with imperfect interface by equivalent inclusion. The last part is the application of the methods and conclusion.

\section{DOUBLY-COATED CIRCLE ASSEMBLAGE MODEL}

Let us consider a representative volume element (RVE) of an isotropic threecomponent material that occupies circular region $V$ of Euclidean space in 2 dimensions. The RVE consists of three-component occupying regions $V_{\alpha} \subset V$ of volume fractions $v_{\alpha}$ $(\alpha=1, \ldots, 3$; the volume of $V$ is assumed to be the unity) and having isotropic conductivities $c_{\alpha}$. A generalization of the Hashin-Shtrikman two-phase coated circle assemblage [7] is the 3-component doubly-coated circle assemblage. Here circles of phase 1 are coated with annular plate of phase 2 , which in turn are coated with annular plate of phase 3 , and the relative volume proportions and coating orders of the phases in all three-compound circles are the same. The space of $V$ is entirely filled by such compound circles distributed randomly with diameters varying to infinitely small (Fig. 1).

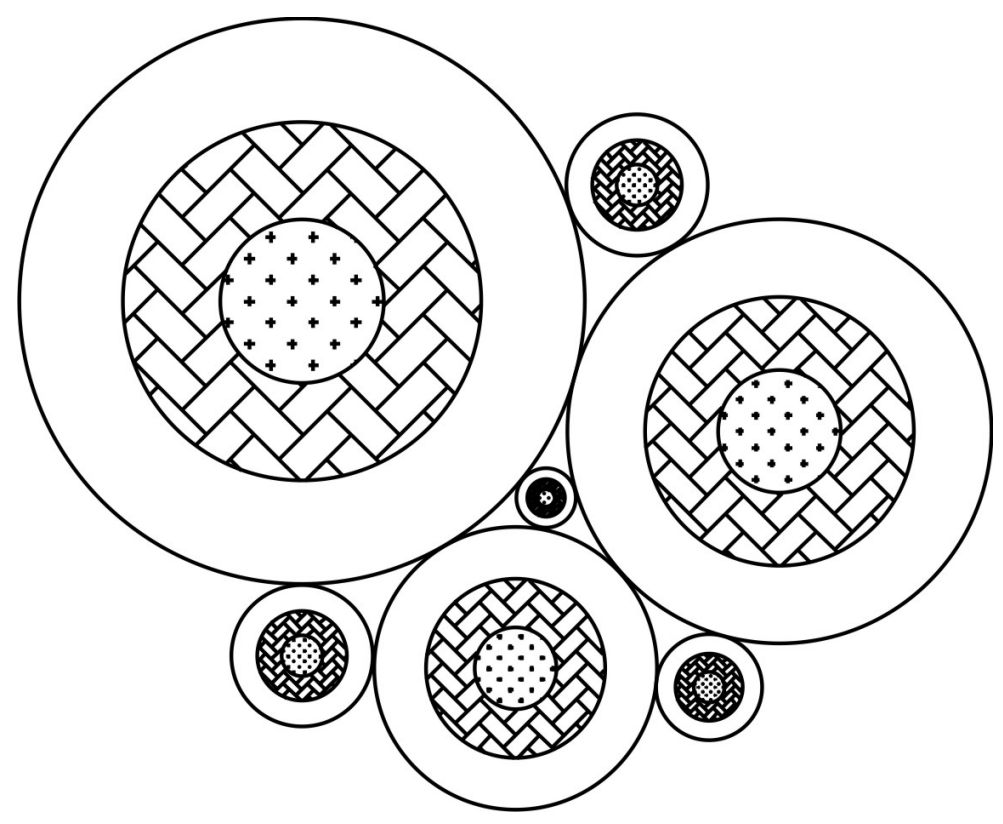

Fig. 1. Doubly-coated circle assemblage

From the minimum energy and complementary energy principles, three-point correlation upper and lower bounds on the effective conductivity of isotropic three-component materials have been constructed $[8,9]$

$$
\left(c_{R}^{-1}-\overline{\mathbf{v}}_{c}^{\prime} \cdot \mathcal{A}_{c}^{-1} \cdot \overline{\mathbf{v}}_{c}\right)^{-1} \leq c^{e f f} \leq c_{V}-\mathbf{v}_{c}^{\prime} \cdot \mathcal{A}_{c}^{-1} \cdot \mathbf{v}_{c}
$$


where

$$
\begin{aligned}
& \mathbf{v}_{c}=\left\{v_{1}\left(c_{1}-c_{R}\right),\left(c_{2}-c_{R}\right), v_{3}\left(c_{3}-c_{R}\right)\right\}^{T}, \quad c_{R}=\left(\sum_{\alpha=1}^{n} v_{\alpha} c_{\alpha}^{-1}\right)^{-1}, \\
& \mathbf{v}_{c}^{\prime}=\left\{\frac{1}{2} v_{1} c_{1}, \cdots, \frac{1}{2} v_{n} c_{n}\right\}^{T}, \quad c_{V}=\sum_{\alpha=1}^{n} v_{\alpha} c_{\alpha}, \\
& \mathcal{A}_{c}=\left\{\mathcal{A}_{\alpha \beta}^{c}\right\}, \quad \mathcal{A}_{\alpha \beta}^{c}=\frac{1}{2} v_{\alpha} c_{\alpha} \delta_{\alpha \beta}+\sum_{\gamma=1}^{n}\left(A_{\gamma}^{\alpha \beta}-v_{\alpha} c_{R} \sum_{\delta=1}^{n} c_{\delta}^{-1} A_{\gamma}^{\delta \beta}\right) c_{\gamma}, \\
& \overline{\mathbf{v}}_{c}=\left\{-v_{1}\left(c_{1}^{-1}-c_{V}^{-1}\right), \ldots,-v_{n}\left(c_{n}^{-1}-c_{V}^{-1}\right)\right\}^{T}, \\
& \overline{\mathbf{v}}_{c}^{\prime}=\left\{-\frac{1}{2} v_{1} c_{1}^{-1}, \ldots,-\frac{1}{2} v_{n} c_{n}^{-1}\right\}^{T}, \\
& \overline{\mathcal{A}}_{c}=\left\{\overline{\mathcal{A}}_{\alpha \beta}^{c}\right\}, \quad \overline{\mathcal{A}}_{\alpha \beta}^{c}=\frac{1}{2} v_{\alpha} c_{\alpha}^{-1} \delta_{\alpha \beta}+\sum_{\gamma=1}^{n}\left(A_{\gamma}^{\alpha \beta}-v_{\alpha} c_{V}^{-1} \sum_{\delta=1}^{n} c_{\delta} A_{\gamma}^{\delta \beta}\right) c_{\gamma}^{-1} .
\end{aligned}
$$

$n$ is number of phases, $\delta_{\alpha \beta}$ is Kronecker symbol and $A_{\gamma}^{\alpha \beta}$ are the three-point correlation parameters.

For the doubly-coated circle assemblage model, these bounds converge to yield the exact value of the effective conductivity [10]

$$
c^{e f f}=c_{V}-\mathbf{v}_{c}^{\prime} \cdot \mathcal{A}_{c}^{-1} \cdot \mathbf{v}_{c} .
$$

In the two-component case, (3) can be presented as

$$
c^{e f f}=P\left(v_{1}, c_{1}, c_{2}\right)=\left(\frac{v_{1}}{c_{1}+c_{2}}+\frac{v_{2}}{2 c_{2}}\right)^{-1}-c_{2},
$$

in which $v_{1}, c_{1}$ are the volume fraction and conductivity of the inclusion, while $v_{2}, c_{2}$ are the volume fraction and conductivity of the matrix. If $v_{2} \ll 1$ (thin coating), one obtains asymptotically expression [11]

$$
c^{e f f}=c_{1}+v_{2} \frac{\left(c_{2}-c_{1}\right)\left(c_{1}+c_{2}\right)}{2 c_{2}}+O\left(v_{2}^{2}\right)
$$

\section{CIRCLE WITH INTERFACIAL THERMAL RESISTANCE}

Consider the two-component coated circle assemblage with thin coating $\left(v_{2} \ll 1\right)$. With the thin coating thickness $h\left(\frac{h}{R_{1}} \ll 1, R_{1}\right.$ is the radii of inner circle) one has

$$
\frac{1}{v_{1}}=\frac{1}{1-v_{2}}=1+v_{2}+O\left(v_{2}^{2}\right)
$$


further

$$
\frac{1}{v_{1}}=\frac{R_{2}^{2}}{R_{1}^{2}}=\left(1+\frac{h}{R_{1}}\right)^{2}=1+\frac{2 h}{R_{1}}+O\left(\frac{h^{2}}{R_{1}^{2}}\right)
$$

hence

$$
v_{2}=\frac{2 h}{R_{1}}+O\left(\frac{h^{2}}{R_{1}^{2}}\right) .
$$

In the lowly conducting imperfect interface model [12], it is assumed that

$$
c_{2}=\frac{h}{\alpha_{K}}, \quad \frac{h}{R_{1}} \rightarrow 0,
$$

where $\alpha_{K}$ is called the Kapitza thermal resistance.

Substituting (8) and (9) into (5), one derives the asymptotic expression of the effective conductivity $c^{1 L}$ of the assemblage of circular inclusions of phase 1 coated by the infinitely thin shells of thermal resistance $\alpha_{K}$

$$
c^{1 L}=\frac{c_{1}}{1+\frac{c_{1} \alpha_{K}}{R_{1}}}+O\left(\frac{h}{R_{1}}\right) .
$$

If these coated inclusions are embedded in the circular shells of the matrix phase $M$, one obtains the effective conductivity of the circle assemblage with the imperfect interface of Kapitza thermal resistance $\alpha_{K}$ (in the limit $h \rightarrow 0$ ) between the inclusion 1 and matrix $M$ components (CAK) by replacing $c^{1 L}$ for $c_{1}$ in (4)

$$
c^{e f f}=P\left(v_{1}, c^{1 L}, c_{M}\right)=\left[\frac{v_{1}}{c_{1}\left(1+c_{1} \alpha_{K} / R_{1}\right)^{-1}+c_{M}}+\frac{v_{M}}{2 c_{M}}\right]^{-1}-c_{M} .
$$

\section{FFT SIMULATION FOR COMPOSITE WITH KAPITZA THERMAL RESISTANCES}

Consider a composite reinforced by fibers aligned in the direction $O x_{3}$. The fibers are regularly distributed in the directions $O x_{1}$ and $O x_{2}$. The contact between fibers and matrix is the imperfect interface of Kapitza's type. This problem can be modelled as a composite of three-component (see for instance [12,13]): inclusion $\left(v_{1}, c_{1}\right)$, matrix $\left(v_{M}, c_{M}\right)$ and interphase with volume fraction $v_{2}=\frac{2 h}{R_{1}}$, conductivity $c_{2}=\frac{h}{\alpha_{K}}$ (Fig. 2 (left)). By substitution scheme, one can consider the two-component composite with perfect interface (Fig. 2 (right)) in which the conductivity of equivalent inclusion is determined by (10)

$$
c_{E I}=c^{1 L}=\frac{c_{1}}{1+\frac{c_{1} \alpha_{K}}{R_{1}}} .
$$

The algorithm for determining the effective conductivity of two-phase periodic materials based on the Fourier transform method (FFT) has been introduced in the literature [14]. 
In the next section, this method will be applied to calculate the effective conductivity of the composite with Kapitza thermal resistance.

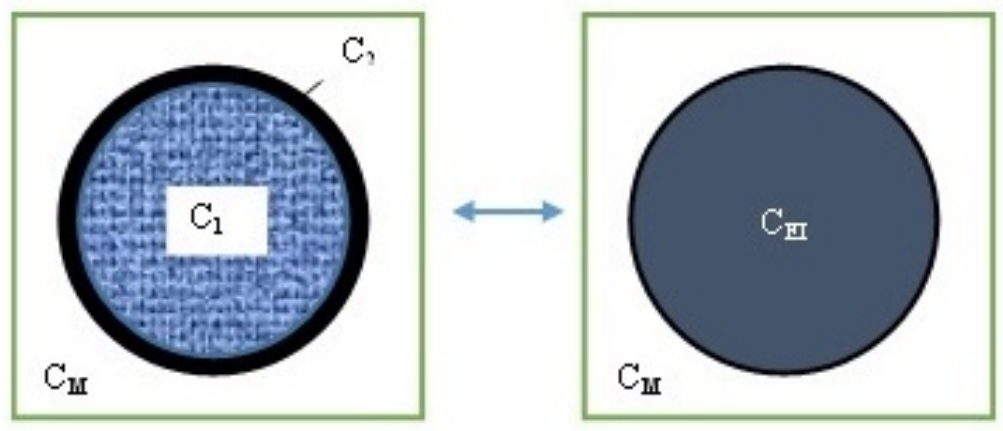

Fig. 2. Unit cell: coated circle assemblage regularly distributed (left), equivalent inclusion (right)

\section{NUMERICAL CALCULATION AND COMPARISON}

We apply these approaches to the case of a composite with imperfect interface of Kapitza's type. Conductivity of the matrix is assumed to be unity $\left(c_{M}=1\right)$, the contrast $c_{1} / c_{M}=10$ and 100. Consider a unit cell having the dimension $L=1$ along each space directions containing inclusion with the dimensionless radius $\left(R_{1} / L\right)$ varies from 0.1 to 0.5. Thermal resistance $\alpha_{K}=0.2 ; 0.5$ and 1 (Tabs. 1-3). For the model of circle with interfacial thermal resistance, the effective conductivity is calculated from (11). The results of FFT simulation is obtained from algorithm in [14] in which the conductivity of the equivalent inclusion is determined from (12). The results of Vincent presented in [15] are also included for comparison.

Table 1. Effective conductivity of composite with imperfect interface of Kapitza's type, $\alpha_{K}=0.2$

\begin{tabular}{ccccccc}
\hline \multicolumn{3}{c}{$c_{1} / c_{M}=10$} & \multicolumn{3}{c}{$c_{1} / c_{M}=100$} \\
\hline$v_{1}$ & CAK & FFT & Vincent [15] & CAK & FFT & Vincent [15] \\
\hline 0.0314 & 0.9780 & 0.9780 & 0.9783 & 0.9791 & 0.9792 & 0.9796 \\
0.0707 & 0.9751 & 0.9752 & 0.9754 & 0.9795 & 0.9795 & 0.9797 \\
0.1257 & 0.9881 & 0.9881 & 0.9881 & 0.9988 & 0.9988 & 0.9988 \\
0.1963 & 1.0209 & 1.0209 & 1.0207 & 1.0421 & 1.0421 & 1.0419 \\
0.2827 & 1.0776 & 1.0776 & 1.0773 & 1.1153 & 1.1155 & 1.1151 \\
0.3848 & 1.1637 & 1.1638 & 1.1634 & 1.2268 & 1.2271 & 1.2267 \\
0.5027 & 1.2874 & 1.2881 & 1.2876 & 1.3898 & 1.3912 & 1.3907 \\
0.6362 & 1.4620 & 1.4649 & 1.4643 & 1.6268 & 1.6336 & 1.6330 \\
0.7543 & 1.6525 & 1.6627 & 1.6620 & 1.8963 & 1.9210 & 1.9204 \\
\hline
\end{tabular}


Table 2. Effective conductivity of composite with imperfect interface of Kapitza's type, $\alpha_{K}=0.5$

\begin{tabular}{ccccccc}
\hline \multicolumn{3}{c}{$c_{1} / c_{M}=10$} & \multicolumn{3}{c}{$c_{1} / c_{M}=100$} \\
\hline$v_{1}$ & CAK & FFT & Vincent [15] & CAK & FFT & Vincent [15] \\
\hline 0.0314 & 0.9586 & 0.9588 & 0.9595 & 0.9589 & 0.9591 & 0.9599 \\
0.0707 & 0.9253 & 0.9255 & 0.9260 & 0.9265 & 0.9266 & 0.9275 \\
0.1257 & 0.8942 & 0.8943 & 0.8948 & 0.8974 & 0.8975 & 0.8983 \\
0.1963 & 0.8697 & 0.8698 & 0.8699 & 0.8764 & 0.8765 & 0.8770 \\
0.2827 & 0.8547 & 0.8547 & 0.8548 & 0.8666 & 0.8666 & 0.8670 \\
0.3848 & 0.8511 & 0.8511 & 0.8510 & 0.8705 & 0.8706 & 0.8707 \\
0.5027 & 0.8607 & 0.8607 & 0.8606 & 0.8907 & 0.8907 & 0.8907 \\
0.6362 & 0.8855 & 0.8854 & 0.8852 & 0.9299 & 0.9299 & 0.9297 \\
0.7543 & 0.9179 & 0.9178 & 0.9175 & 0.9777 & 0.9777 & 0.9775 \\
\hline
\end{tabular}

Table 3. Effective conductivity of composite with imperfect interface of Kapitza's type, $\alpha_{K}=1$

\begin{tabular}{ccccccc}
\hline \multicolumn{3}{c}{$c_{1} / c_{M}=10$} & \multicolumn{3}{c}{$c_{1} / c_{M}=100$} \\
\hline$v_{1}$ & CAK & FFT & Vincent [15] & CAK & FFT & Vincent [15] \\
\hline 0.0314 & 0.9498 & 0.9499 & 0.9516 & 0.9499 & 0.9500 & 0.9523 \\
0.0707 & 0.9003 & 0.9004 & 0.9014 & 0.9007 & 0.9008 & 0.9026 \\
0.1257 & 0.8442 & 0.8444 & 0.8454 & 0.8453 & 0.8454 & 0.8471 \\
0.1963 & 0.7868 & 0.7869 & 0.7877 & 0.7890 & 0.7891 & 0.7899 \\
0.2827 & 0.7313 & 0.7313 & 0.7318 & 0.7353 & 0.7353 & 0.7360 \\
0.3848 & 0.6802 & 0.6799 & 0.6799 & 0.6866 & 0.6863 & 0.6868 \\
0.5027 & 0.6348 & 0.6338 & 0.6336 & 0.6444 & 0.6435 & 0.6438 \\
0.6362 & 0.5959 & 0.5935 & 0.5930 & 0.6096 & 0.6074 & 0.6076 \\
0.7543 & 0.5699 & 0.5654 & 0.5650 & 0.5875 & 0.5836 & 0.5837 \\
\hline
\end{tabular}

Figs. 3-8 presents the effective conductivity of composite as function of the volume fraction $v_{1}$ for various values of $\alpha_{K}$ and ratio $c_{1} / c_{M}$. One can see a good agreement between the circle assemblage, the FFT simulation and Vincent's results [15].

From these figures, one also see the dependence of the effective conductivity on the Kapitza thermal resistance. When $\alpha_{K}=0.2$, one observes a slight decrease then increase. When $\alpha_{K}=0.5$, three curves decrease, reach a minimum at $v_{1}=0.3$ or 0.4 and then increase. This illustrates the effect of inclusion and interface on the effective conductivity. The inclusion with $c_{1}>c_{M}$ increases the effective conductivity while the thermal discontinuity at the interface between inclusion and matrix decreases the effective conductivity. When $\alpha_{K}=1$, the curves decrease for all values of volume fraction $v_{1}$. This shows the dominance of the thermal resistance coefficient for the effective conductivity. 


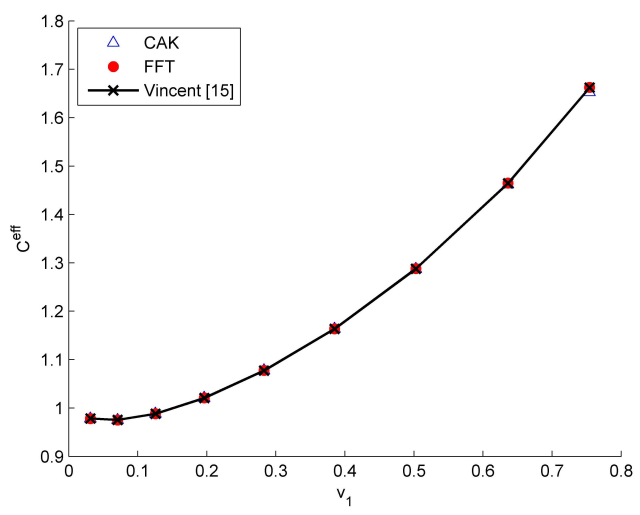

Fig. 3. Effective conductivity of composite with imperfect interface of Kapitza's type, $\alpha_{K}=0.2 ;$ contrast $c_{1} / c_{M}=10$

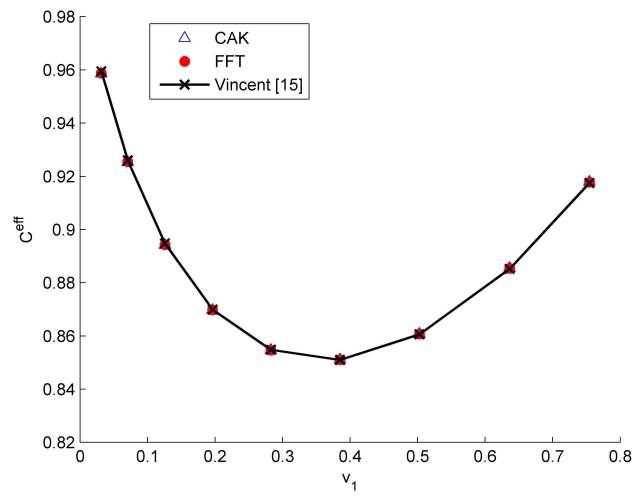

Fig. 5. Effective conductivity of composite with imperfect interface of Kapitza's type, $\alpha_{K}=0.5 ;$ contrast $c_{1} / c_{M}=10$

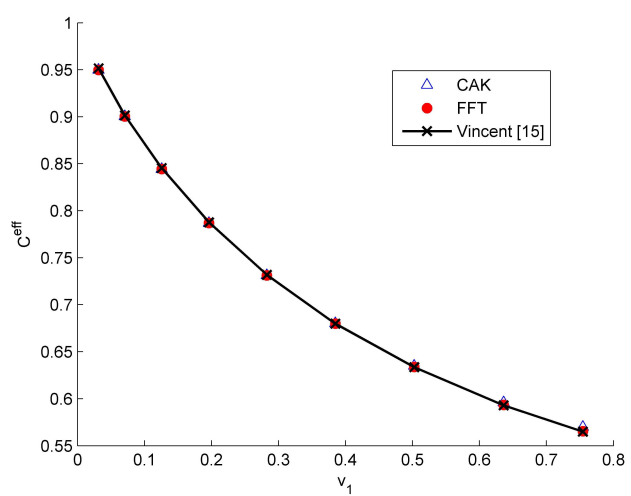

Fig. 7. Effective conductivity of composite with imperfect interface of Kapitza's type, $\alpha_{K}=1$; contrast $c_{1} / c_{M}=10$

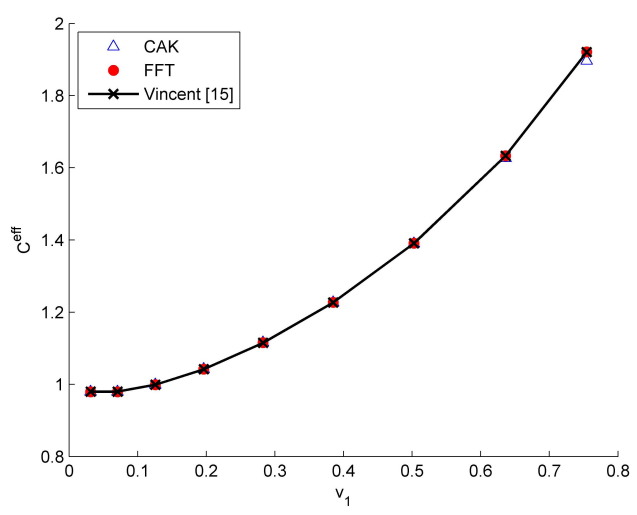

Fig. 4. Effective conductivity of composite with imperfect interface of Kapitza's type, $\alpha_{K}=0.2$; contrast $c_{1} / c_{M}=100$

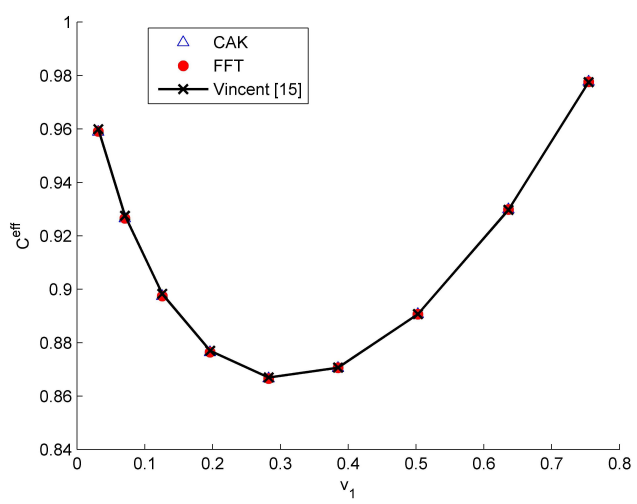

Fig. 6. Effective conductivity of composite with imperfect interface of Kapitza's type, $\alpha_{K}=0.5 ;$ contrast $c_{1} / c_{M}=100$

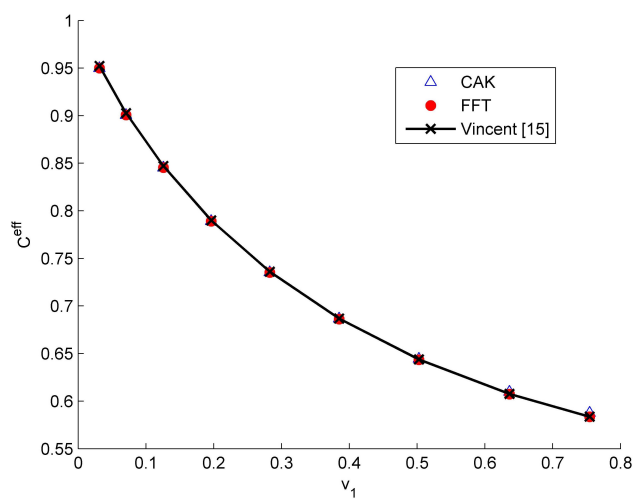

Fig. 8. Effective conductivity of composite with imperfect interface of Kapitza's type, $\alpha_{K}=1$; contrast $c_{1} / c_{M}=100$ 


\section{CONCLUSION}

The paper presents the methods to compute the effective conductivity of composites with imperfect interfaces of Kapitza's type. In this case, the temperature is discontinuous while the flux remaining continuous across the interface. First approach is based on the model of circle assemblage with thin coating. Second approach is based on the FFT simulation combining equivalent-inclusion approach. The results show that the circle assemblage models give good approach when simulating the effective conductivity of composite with imperfect interface. Determination of the effective conductivity of composite with imperfect interface was also solved by Vincent [15]. In order to simulate the discontinuities of the temperature, an enrichment of the space around the interface is introduced. An additional temperature field which are null outside of the inclusion but are different of zero inside the inclusion is added. This method gives accurate results but requires more time calculations and computer memory. With the equivalent-inclusion approach, the FFT simulation in this paper provides a simpler method for computing the effective conductivity while still ensuring accuracy. The approach shows the ability to account for the size-dependency of the effective conductivity of the composite with the radius of the inclusion. The application of the method to three-dimensional problems is in progress.

\section{ACKNOWLEDGMENT}

This research is supported by Vietnam National Foundation for Science and Technology Development (NAFOSTED) under grant number 107.02-2018.15.

\section{REFERENCES}

[1] P. L. Kapitza. The study of heat transfer in helium II. J. Phys. (USSR), 4, (1941), pp. 181-210.

[2] H. Hatta and M. Taya. Thermal conductivity of coated filler composites. Journal of Applied Physics, 59, (6), (1986), pp. 1851-1860. https://doi.org/10.1063/1.336412.

[3] Y. Benveniste and T. Miloh. The effective conductivity of composites with imperfect thermal contact at constituent interfaces. International Journal of Engineering Science, 24, (9), (1986), pp. 1537-1552. https://doi.org/10.1016/0020-7225(86)90162-x.

[4] D. P. H. Hasselman and L. F. Johnson. Effective thermal conductivity of composites with interfacial thermal barrier resistance. Journal of Composite Materials, 21, (6), (1987), pp. 508515. https://doi.org/10.1177/002199838702100602.

[5] M. L. Dunn and M. Taya. The effective thermal conductivity of composites with coated reinforcement and the application to imperfect interfaces. Journal of Applied Physics, 73, (4), (1993), pp. 1711-1722. https://doi.org/10.1063/1.353206.

[6] S. Torquato and M. D. Rintoul. Effect of the interface on the properties of composite media. Physical Review Letters, 75, (22), (1995), p. 4067. https://doi.org/10.1103/physrevlett.76.3241.

[7] Z. Hashin and S. Shtrikman. A variational approach to the theory of the effective magnetic permeability of multiphase materials. Journal of Applied Physics, 33, (10), (1962), pp. 31253131. https://doi.org/10.1063/1.1728579.

[8] D. C. Pham, L. D. Vu, and V. L. Nguyen. Bounds on the ranges of the conductive and elastic properties of randomly inhomogeneous materials. Philosophical Magazine, 93, (18), (2013), pp. 2229-2249. https://doi.org/10.1080/14786435.2013.765992. 
[9] T. K. Nguyen, V. L. Nguyen, and D. C. Pham. Estimating effective conductivity of unidirectional transversely isotropic composites. Vietnam Journal of Mechanics, 35, (3), (2013), pp. 203213. https://doi.org/10.15625/0866-7136/35/3/2767.

[10] D. C. Pham. Bounds on the effective conductivity of statistically isotropic multicomponent materials and random cell polycrystals. Journal of the Mechanics and Physics of Solids, 59, (3), (2011), pp. 497-510. https://doi.org/10.1016/j.jmps.2011.01.006.

[11] D. C. Pham. Solutions for the conductivity of multi-coated spheres and spherically symmetric inclusion problems. Zeitschrift Für Angewandte Mathematik Und Physik, 69, (2018). https://doi.org/10.1007/s00033-017-0905-6.

[12] H. Le Quang, D. C. Pham, G. Bonnet, and Q.-C. He. Estimations of the effective conductivity of anisotropic multiphase composites with imperfect interfaces. International Journal of Heat and Mass Transfer, 58, (1-2), (2013), pp. 175-187. https://doi.org/10.1016/j.ijheatmasstransfer.2012.11.028.

[13] Z. Hashin. Thin interphase/imperfect interface in conduction. Journal of Applied Physics, 89, (4), (2001), pp. 2261-2267. https://doi.org/10.1063/1.1337936.

[14] V. L. Nguyen and T. K. Nguyen. FFT simulations and multi-coated inclusion model for macroscopic conductivity of 2D suspensions of compound inclusions. Vietnam Journal of Mechanics, 37, (3), (2015), pp. 169-176. https://doi.org/10.15625/0866-7136/37/3/5096.

[15] V. Monchiet. FFT based iterative schemes for composites conductors with non-overlapping fibers and Kapitza interface resistance. International Journal of Solids and Structures, 135, (2018), pp. 14-25. https://doi.org/10.1016/j.ijsolstr.2017.10.015. 